\title{
BASES TEÓRICAS DE LA INVESTIGACIÓN CUALITATIVA EN CIENCIAS CONTABLES
}

\author{
THEORETICAL BASES OF QUALITATIVE RESEARCH IN ACCOUNTING SCIENCES
}

\section{RESUMEN}

El enfoque cualitativo en las investigaciones de las Ciencias Contables se fundamenta en un proceso de investigación que permite alcanzar resultados diferentes a los del método cuantitativo. El objetivo general de la investigación fue describir las principales características de las bases teóricas de la investigación cualitativa en Ciencias Contables. Se realizó un análisis documental de 20 artículos científicos de referentes en las Ciencias Contables sobre la investigación cualitativa. Los resultados fueron procesados con Atlas. Ti. y mostrados en forma narrativa y conceptual. Los principales resultados fueron: 1) Existen variadas posibilidades de poder llevar a cabo una investigación cualitativa y 2) El enfoque cualitativo de las investigaciones permite ser más abierto, resolver problemas que no se pudieron abordar con otro enfoque y cuestionar aspectos de la investigación cuantitativa. La principal conclusión fue: Los resultados de las investigaciones cualitativas permiten utilizarlas como herramienta complementaria a los estados financieros, por ser una manera diferente de entender el proceso contable.

Palabras clave: Ciencias Contables; enfoque cualitativo; investigación cualitativa; enfoque cuantitativo; método cuali-cuanti.

\author{
Miguel Ángel Alegre Brítez \\ Universidad Nacional de Asunción \\ Asunción, Paraguay \\ ORCID: https://orcid.org/0000-0003-4265-9391 \\ Correo electrónico: malegre@eco.una.py \\ Chap Kau Kwan Chung \\ Universidad del Pacífico \\ Asunción, Paraguay \\ ORCID: https://orcid.org/0000-0002-5478-3659 \\ Correo electrónico:wendy505@hotmail.com
}

\begin{abstract}
The qualitative approach in the investigations of the Accounting Sciences is based on a research process that allows achieving results that are different from those of the quantitative method. The research's general objective was to describe the main characteristics of the qualitative research's theoretical bases in accounting sciences. A documentary analysis of 20 scientific articles of referents in the accounting sciences on qualitative research was carried out. The results were processed through Atlas. You. and shown in a narrative and conceptual form. The main results were: 1) There are various possibilities to carry out a qualitative research and 2) The qualitative approach of the investigations allows to be more open, to solve problems that could not be addressed with a different approach, and to question aspects of the quantitative research. The main conclusion was: The results of the qualitative research allow them to be used as a complementary tool to financial statements, as they are a different way of understanding the accounting process.
\end{abstract}

Keywords: Accounting sciences; qualitative approach; qualitative research; quantitative approach; quali-quanti method.

(c) Los autores. Este artículo es publicado por la revista Quipukamayoc de la Facultad de Ciencias Contables, Universidad Nacional Mayor de San Marcos. Este es un artículo de acceso abierto, distribuido bajo los términos de la licencia Creative Commons Atribución 4.0 Internacional (CC BY 4.0) [https://creativecommons.org/licenses/ by/4.0/deed.es] que permite el uso, distribución y reproducción en cualquier medio, siempre que la obra original sea debidamente citada de su fuente original. 


\section{INTRODUCCIÓN}

En el artículo se aborda la teoría de la investigación cualitativa como una de las nuevas formas en que la ciencia se manifiesta frente a las necesidades humanas (Moscoso Córdova, 2019). La característica principal de lo cualitativo es su base teórica, por lo que busca comprender los fenómenos sociales y culturales a través de un procesamiento sensorial y emocional (Ayuso Moya y Ripoll Feliu, 2005). Por otra parte, el objetivo de la investigación cualitativa puede ser descriptivo (socio-cultural), etnográfico o histórico, aunque también es posible destinarla a fines específicos como el diagnóstico, entrevista y revisión documental (Pérez Stizzoli, 2014). La forma más corriente de este tipo de investigación son los estudios evaluativos, cuya finalidad es conocer las realidades sociales desde un enfoque cualitativo (Quintero Dávila, 2012). La teoría contable subyacente a la investigación cualitativa en las Ciencias Contables puede llevarse a cabo en dos situaciones: 1) cuando se conoce y analiza el origen de una idea y 2) como elemento fundamental para suplir lagunas metodológicas del método cuantitativo. Esto permite que la investigación cualitativa en Ciencias Contables pueda desarrollarse como una forma de entender el proceso contable para realizar análisis estratégicos y contextualizar el proceso de revisión financiera. Por lo cual, el artículo trata de describir algunas formas de realizar esta investigación cualitativa como recurso complementario.

La investigación cualitativa puede ser considerada como una opción valiosa para el entendimiento del mundo cultural y social a partir de la relación subjetiva que se establece entre los sujetos participantes en la misma (Beltrán Beltrán y González Feliciano, 2017). Para que una investigación contable sea cualitativa, es importante recordar que el objetivo fundamental de este tipo de investigación es conocer mejor a los sujetos participantes, sus mundos y las realidades sociales en la que se insertan (Hernández Rodríguez y Escobar Castillo, 2017). Para ello, se valora la cualidad de una relación sobre la base del interés profesional y no por el bienestar o satisfacción del individuo (Bolzan, Vendruscolo, Sallaberry y Diehl, 2020). Las Ciencias Contables han tenido que cambiar en cuanto al análisis de los sujetos sociales, culturales y organizacionales, en las nuevas relaciones con sus contextos (Ospina Zapata, 2005). La investigación en contabilidad no se puede realizar solo desde una perspectiva científica y positivista, ya que en estos casos es fundamental la aplicación de los métodos cualitativos para comprender mejor la investigación y sus procesos (Chango Galarza, 2019). Sin embargo, se debe tener presente que la aplicación de este tipo de métodos en la disciplina no siempre puede ser fácil de usar (Ospina Delgado, 2018). Para ello es importante identificar la teoría y el tipo de método que se va a utilizar para dar validez a la investigación (Monsalve, 2006).

En este trabajo, se aborda las bases teóricas de la investigación cualitativa en contabilidad, así como los tipos de métodos aplicables. Se plantea el valor que puede adquirir la investigación cualitativa dentro de la contabilidad y las implicaciones para el desempeño profesional del individuo. Por lo expuesto, la pregunta general de esta investigación es: ¿Cuáles son las principales características de las bases teóricas de la investigación cualitativa en Ciencias Contables? Se tiene como objetivo general el: Describir las principales características de las bases teóricas de la investigación cualitativa en Ciencias Contables. Como hipótesis se propone que las Ciencias Contables tienen bases teóricas en su constitución y desarrollo. En cuanto a las limitaciones del estudio, se tuvo la escasa bibliografía concerniente a la teoría contable en el ámbito de Latinoamérica.

El enfoque fue cualitativo y el diseño de la investigación fue narrativo, puesto que se describieron las características en común de los artículos científicos con las teorías contables. Los métodos de investigación fueron el inductivo y analítico, ya que se partió de casos particulares para llegar a un análisis general detallado. Se realizó una revisión bibliográfica en Google Académico de 20 artículos científicos de referentes sobre temas de investigación cualitativa en las Ciencias Contables durante el periodo 20002020, para lo cual se utilizaron las siguientes palabras clave de búsqueda: "investigación cualitativa", "enfoque cualitativo", "métodos de investigación cualitativos", "contabilidad", "ciencias contables". El procesamiento se hizo mediante el programa Atlas. Ti., donde se introdujo el texto completo, lo que ayudó a establecer aquellas categorías de análisis tales como teoría contable, bases teóricas, contabilidad, y la ciencia contable a efectos de su condensación y análisis. Los resultados fueron presentados en forma de tablas con narrativas.

\section{ALCANCE DE LA REVISIÓN}

En este estudio, se analizaron los aspectos básicos de la investigación cualitativa en las Ciencias Contables. Los artículos analizados se presentan en la Tabla 1.

La investigación cualitativa en las Ciencias Contables surge de una reacción crítica contra el llamado paradigma positivista que imperaba durante el siglo XX (Báez Hernández, 2015), por lo cual, generalmente se considera que la investigación es un proceso más que un producto (Escobar Pérez y Lobo Gallardo, 2014).

Como objetivo se espera que la investigación cualitativa sea una investigación crítica (Barona, 2020); es por ello 
Tabla 1

Artículos analizados

\section{Artículos analizados con Atlas.ti}

Arias Collaguazo, W. M., Maldonado Gudiño, C. W., \& Arciniegas Paspuel, O. G. (2021). Tendencias epistemológicas de las ciencias contables en titulaciones de pregrado de las universidades ecuatorianas. Universidad y Sociedad, 13(3), 354-360. Recuperado de https://rus.ucf.edu.cu/index.php/rus/article/view/2106

Arriaga Baidal, G. C., Reyes Tomalá, M. V., Olives Maldonado, J. C., \& Solórzano Méndez, V. (2017). Análisis de la cultura tributaria: Impuesto a la Renta para Personas Naturales No Obligadas a llevar Contabilidad, Provincia de Santa Elena. Revista Ciencias Pedagógicas e Innovación, 5(3), 118-127. https://doi.org/10.26423/rcpi.v5i3.214

Ayuso Moya, A., \& Ripoll Feliu, V. (2005). El estudio de casos como prototipo de la investigación en contabilidad de gestión desde una perspectiva cualitativa. Revista Iberoamericana de Contabilidad de Gestión, 131-168. Recuperado de http://www.observatorio-iberoamericano.org/RICG/N\%C2\%BA 5/Amparo\%20Ayuso\%20Moya\%20y\%20Vicente\%20Ripoll\%20Feliu.pdf

Báez Hernández, A. (2015). Consideraciones sobre la implementación de un Sistema de Investigación Científica en las Ciencias Contables. Revista Publicando, 2(3), 61-89. Recuperado de https://dialnet.unirioja.es/servlet/articulo?codigo=5833495

Barona, N. (2020). Evolución y nuevas tendencias de la investigación contable. Actualidad Contable Faces, 23(40), 9-27. Recuperado de https://www.redalyc.org/jatsRepo/257/25763378002/25763378002.pdf

Beltrán Beltrán, J. P., \& González Feliciano, L. A. (2017). Tendencia de investigación contable en Colombia: Teoría o práctica, una mirada desde las Revistas Contables Colombianas 2011 a 2015. FACE: Revista de la Facultad de Ciencias Económicas y Empresariales, 17(2), 68-80. https://doi.org/10.24054/01204211.v2.n2.2017.2228

Bolzan, G., Vendruscolo, M. I., Sallaberry, J. D., \& Diehl, W. (2020). Enseñanza de ciencias contables y el proceso de convergencia a las international financial reporting standards. Revista Ambiente Contábil - Universidade Federal do Rio Grande do Norte - ISSN 2176-9036, 12(1). https://doi.org/10.21680/2176-9036.2020v12n1ID19618

Cárdenas Londoño, R., Mesa Velásquez, G. S., \& Restrepo Duque, D. F. (2020). Análisis de la producción científica y académica de los miembros de Redsicon: Estudio de caso. Revista Visión Contable, (21), 121-141. https://doi.org/10.24142/rvc.n21a6

Chango Galarza, M. (2019). El problema metodológico de la investigación contable: Una fundamentación basada en los enfoques positivista e interpretativo. Prospectivas UTC «Revista de Ciencias Administrativas y Económicas», 2(2), 142-151. Recuperado de http://investigacion.utc.edu.ec/revistasutc/index.php/prospectivasutc/article/view/273

Charres, H. (2018). Triangulación: Una estrategia adecuada para las investigaciones en las ciencias administrativas y contables en la educación superior. Acción y Reflexión Educativa, (43), 152-165. Recuperado de https://www.revistas.up.ac.pa/index.php/ accion reflexion_educativa/article/view/565

Escobar Pérez, B., \& Lobo Gallardo, A. (2014). Implicaciones Teóricas y Metodológicas de la Evolución de la Investigación en Contabilidad de Gestión. Spanish Journal of Finance and Accounting / Revista Española de Financiación y Contabilidad. (world). Recuperado de https://www.tandfonline.com/doi/abs/10.1080/02102412.2002.10779450

González-Díaz, R. R., \& Serrano Polo, E. A. (2018). Entrevistas Espontaneas Categoriales (EEC) para la construcción de categorías orientadoras en la investigación cualitativa. Journal of Latin American Science, 2(1), 1-11. Recuperado de https://lasjournal.com/ index.php/abstract/article/view/2

Gutiérrez Huby, A. M. (2005). Importancia de los indicadores en la autoevaluación de la Facultad de Ciencias Contables. Quipukamayoc, 12(24), 59-63. https://doi.org/10.15381/quipu.v12i24.5442

Hernández Rodríguez, D. R., \& Escobar Castillo, A. E. (2017). Modelo de contabilidad social como herramienta de gestión para la responsabilidad social empresarial. IJMSOR: International Journal of Management Science \& Operation Research, 2(1), 44-56. Recuperado de http://ijmsoridi.com/index.php/ijmsor/article/view/86

Mejía Soto, E., Morra Roa, G., \& Montes Salazar, C. A. (2013). Adscripción de la contabilidad en la estructura general del conocimiento. Cuadernos de Contabilidad, 14(34), 159-187. Recuperado de https://dialnet.unirioja.es/servlet/articulo?codigo=5447038

Moscoso Córdova, J. (2019). La investigación cualitativa y la contabilidad: El rol de los estudios en ciencias contables en la construcción de sociedades más justas y equitativas. Prospectivas UTC «Revista de Ciencias Administrativas y Económicas», 2(2), 171-184. Recuperado de http://investigacion.utc.edu.ec/revistasutc/index.php/prospectivasutc/article/view/289

Naranjo Rivera, O. (2009). Aportes para un debate sobre el método de la investigación cualitativa y la investigación contable. Adversia, (4), 105-114. Recuperado de https://revistas.udea.edu.co/index.php/adversia/article/view/2035

Ortíz Sánchez, L. M. (2020). La teoría fundamentada como método de investigación para el desarrollo de la educación contable. Revista Visión Contable, (22), 60-77. https://doi.org/10.24142/rvc.n22a3

Ospina Zapata, C. M. (2005). Sobre la investigación en contabilidad: Algunos apuntes. Contaduría Universidad de Antioquia, (46), 73-110. Recuperado de https://revistas.udea.edu.co/index.php/cont/article/view/25679

Pérez Stizzoli, V. (2014). La investigación cualitativa en contabilidad.: Un análisis a partir de revistas indexadas. CAPIC REVIEW, 12(2), 147-158. Recuperado de https://dialnet.unirioja.es/servlet/articulo?codigo=7145015

Fuente: Elaboración propia con base en el software Atlas.ti 
que se hace un breve repaso sobre los fundamentos teóricos de la investigación cualitativa tanto en ciencias humanas como contables (Ortiz-Ocaña, 2016) (Ver Tablas 2, 3 y 4$)$.

Es importante resaltar que la vida social de una empresa no es un objeto, sino un conjunto de objetos (personas, organizaciones, instituciones, sistemas y tecnologías, etc.) que se relacionan entre sí para producir y transmitir valor a través del tiempo. Del mismo modo, desde esta perspectiva, la empresa no es un objeto, sino un conjunto de objetos que interactúan entre sí. Cabe afirmar, también, que no existe ninguna empresa sola, ya que es una entidad compuesta por diferentes objetos (Pérez y Pinto Perry, 2011). Así, la teoría financiera contable nos proporciona una visión global de la empresa, las finanzas y la contraloría; es decir, los objetos que interactúan entre sí para producir y transmitir valor a través del tiempo.

Por otra parte, el ente que promueve y difunde la cultura de la empresa es el empresario. Este último posee una visión de conjunto de su cuidadosa inclusión en el mundo laboral, ya sea a través del apoyo a los usuarios más desfavorecidos (alimentación, vivienda, etc.) o por medio de la construcción del espíritu solidario y altruista de sus trabajadores. La filosofía teórica contable empresarial promueve la capacitación para rescatar el conocimiento adquirido en los últimos años, lo que ha permitido una importante participación de la actividad empresarial en el contexto de la gestión del conocimiento.

La cultura empresarial se construye a través de normas y prácticas sociales que se transmiten a través del tiempo. De esta manera, se pueden descubrir múltiples elementos que constituyen las bases estructurales de esta cultura empresarial.

La empresa como entidad social se va construyendo desde el momento en que una actividad nueva viene a implantarse en la sociedad. Los nuevos valores y nuevas formas de vida son promovidos por la empresa (Charres, 2018). El proceso de modernización de la sociedad se manifiesta en el procesamiento del trabajo y las relaciones sociales ante el mundo laboral, a través del cual esta última define los objetivos y las normas para la conducta humana (Mejía Soto, Morra Roa y Montes Salazar, 2013).

Tabla 2

Investigación cualitativa

\begin{tabular}{cll}
\hline Categoría & \multicolumn{1}{c}{ Concepto 1 } & \multicolumn{1}{c}{ Concepto 2 } \\
\hline Investigación cualitativa & $\begin{array}{l}\text { La investigación cualitativa no es necesariamente } \\
\text { una investigación aislada (Gutiérrez Huby, 2005). }\end{array}$ & $\begin{array}{l}\text { Es necesario contar con un enfoque conceptual o } \\
\text { metodológico que nos permita entender el objeto de } \\
\text { estudio (Caraguay Narváez, 2016). }\end{array}$ \\
\hline
\end{tabular}

Fuente: Elaboración propia

Tabla 3

Investigación cualitativa en las ciencias contables

\begin{tabular}{|c|c|c|}
\hline Categoría & Concepto 1 & Concepto 2 \\
\hline $\begin{array}{l}\text { Investigación cualitativa } \\
\text { en las ciencias contables }\end{array}$ & $\begin{array}{l}\text { Se menciona la necesidad de desarrollar una inves- } \\
\text { tigación cualitativa en las ciencias contables (Pinto } \\
\text { Perry, 2010). }\end{array}$ & $\begin{array}{l}\text { La investigación de las Ciencias Contables, como } \\
\text { cualquier otra disciplina académica, debe ir encami- } \\
\text { nada a descubrir el conocimiento sobre un objeto a } \\
\text { estudiar (González-Díaz y Serrano Polo, 2018). }\end{array}$ \\
\hline
\end{tabular}

Fuente: Elaboración propia

Tabla 4

Relación entre Ciencia y Ciencias Contables

Ciencia Ciencias Contables

La Ciencia no es un objeto, sino más bien la actividad humana que busca acceder a los objetos, descubrir sus características y determinar su relación con otros (Cárdenas Londoño, Mesa Velásquez y Restrepo Duque, 2020).

En ciencias contables se pueden encarar los aspectos de la investigación con diferentes enfoques (Arias Collaguazo, Maldonado Gudiño y Arciniegas Paspuel, 2021).

Fuente: Elaboración propia 
La relación entre la empresa y la sociedad se establece mediante las Ciencias Contables (Naranjo Rivera, 2009). La investigación contable no es una disciplina estática, ya que evoluciona a lo largo del tiempo, en la medida que se encuentra en constante interacción con el mundo en el que se desarrolla (Arriaga Baidal, Reyes Tomalá, Olives Maldonado y Solórzano Méndez, 2017). La teoría contable pura, que se limita a la simple exposición de los hechos contables, no es suficiente para establecer una filosofía ética contable; pues la investigación contable es una disciplina que surge como una reacción crítica contra el proyecto de ciencias sociales positivistas y, a partir del cual, se manifiesta una acción crítica contra la práctica de la contabilidad.

La investigación contable no es una simple teoría que pretende explicar la evolución histórica de los objetos de estudio. Por ello, es necesario cuestionar a diario los fundamentos teóricos y metodológicos que sustentan la disciplina (Ortíz Sánchez, 2020). En la actualidad, se experimenta en las Ciencias Contables, una visión metodológica que podría ser considerada como una reacción crítica contra el paradigma positivista de las ciencias sociales (Saavedra y Saavedra, 2015).

Esto significa que la investigación cualitativa ha venido a reivindicar los valores de la emoción, del significado emocional del ser humano y de su interacción con el mundo circundante (Villarreal y Córdoba Martínez, 2017). Las Ciencias Contables tienen una epistemología y axiomatización teórica y metodológica que definen su rigurosidad y enfoque crítico; así como una epistemología teórica que permite al profesor investigador tomar decisiones acertadas. Hoy por hoy existe una tendencia académica a la investigación cualitativa en las Ciencias Contables.

El proceso de elaboración de los estados financieros tiene un componente social porque la sociedad productiva y la sociedad de consumo tienen como objetivo las producciones y las actividades destinadas a satisfacer los dictámenes de bienestar, económico y social. Es necesario afirmar que se presenta un proceso de transición entre las cosas dinámicas más abiertas que nunca y el estado del conocimiento.

La investigación cualitativa en las Ciencias Contables no es un mero paso para una investigación descriptiva. El contador tiene una importante labor, ya que es él quien detalla los resultados financieros de la empresa. Debido a que la contabilidad es una contribución al conocimiento del mundo y un proceso de transformación de los conocimientos, la investigación cualitativa exige no solo reflexionar, ver y comprender, sino crear nuevos conocimientos.
Los hallazgos destacables en la literatura latinoamericana son aquellos que abordan la relación intrínseca entre la ciencia, en general, con las Ciencias Contables a partir de fundamentos teóricos y epistemológicos que tienen una base conceptual, pero que carecen de un mayor análisis por parte de los académicos latinoamericanos a efectos de la profundización del objeto de estudio.

\section{CONCLUSIONES}

Como consecuencia de la revisión, se apunta a la necesidad de considerar un enfoque cualitativo en las investigaciones de las Ciencias Contables. Se entiende que el proceso de investigación cualitativa requiere mayor atención en los centros académicos latinoamericanos, ya que es una disciplina emergente $y$, asimismo, desconocida para muchos profesionales contables, puesto que la mayoría de estos solo abordan a las Ciencias Contables desde un punto de vista o enfoque cuantitativo. Un aspecto relevante es la manera en la que se actualiza y desea mejorar esta disciplina, ya que para ello es necesario llevar a cabo investigaciones de tipo cualitativo. Un punto importante es la existencia de herramientas con las que pueda desarrollarse, incluso, el enfoque cualitativo de las investigaciones. En la actualidad, el enfoque cualitativo es tan importante como el enfoque cuantitativo. El hecho de que los resultados sean diferentes no significa que se corra riesgos, sino que existe la posibilidad de buscar metodologías adecuadas a las necesidades y no descartar ninguna investigación.

Como línea de investigación futura, se propone la investigación cualitativa de las Ciencias Contables con base en las Normas Internacionales de Información Financiera y las Normas Internacionales de Contabilidad del Sector Público.

\section{REFERENCIAS}

Arias Collaguazo, W. M., Maldonado Gudiño, C. W., \& Arciniegas Paspuel, O. G. (2021). Tendencias epistemológicas de las ciencias contables en titulaciones de pregrado de las universidades ecuatorianas. Universidad y Sociedad, 13(3), 354-360. Recuperado de: https://rus.ucf.edu.cu/index.php/rus/article/view/2106

Arriaga Baidal, G. C., Reyes Tomalá, M. V., Olives Maldonado, J. C., \& Solórzano Méndez, V. (2017). Análisis de la cultura tributaria: Impuesto a la Renta para Personas Naturales No Obligadas a llevar Contabilidad, Provincia de Santa Elena. Revista Ciencias Pedagógicas e Innovación, 5(3), 118-127. https://doi.org/10.26423/ rcpi.v5i3.214

Ayuso Moya, A., \& Ripoll Feliu, V. (2005). El estudio de casos como prototipo de la investigación en contabilidad 
de gestión desde una perspectiva cualitativa. Revista Iberoamericana de Contabilidad de Gestión, 131-168. Recuperado de: http://www.observatorio-iberoamericano.org/RICG/N\%C2\%BA_5/Amparo\%20Ayuso\%20 Moya\%20y\%20Vicente\%20Ripoll\%20Feliu.pdf

Báez Hernández, A. (2015). Consideraciones sobre la implementación de un Sistema de Investigación Científica en las Ciencias Contables. Revista Publicando, 2(3), 61-89. Recuperado de: https://dialnet.unirioja.es/servlet/articulo? codigo $=5833495$

Barona, N. (2020). Evolución y nuevas tendencias de la investigación contable. Actualidad Contable Faces, 23(40), 9-27. Recuperado de: https://www.redalyc.org/ jatsRepo/257/25763378002/25763378002.pdf

Beltrán Beltrán, J. P., \& González Feliciano, L. A. (2017). Tendencia de investigación contable en Colombia: Teoría o práctica, una mirada desde las Revistas Contables Colombianas 2011 a 2015. FACE: Revista de la Facultad de Ciencias Económicas y Empresariales, 17(2), 68-80. https://doi.org/10.24054/01204211. v2.n2.2017.2228

Bolzan, G., Vendruscolo, M. I., Sallaberry, J. D., \& Diehl, W. (2020). Enseñanza de ciencias contables y el proceso de convergencia a las international financial reporting standards. Revista Ambiente Contábil - Universidade Federal do Rio Grande do Norte - ISSN 2176-9036, 12(1). https://doi.org/10.21680/2176-9036.2020v12n1ID19618

Caraguay Narváez, M. J. (2016). Consideraciones generales para la formulación de un problema de investigación científica aplicadas en las ciencias contables (Tesis de grado en contabilidad). Universidad Técnica de Machala, Machala. Recuperado de: http://repositorio.utmachala.edu.ec/handle/48000/8706

Cárdenas Londoño, R., Mesa Velásquez, G. S., \& Restrepo Duque, D. F. (2020). Análisis de la producción científica y académica de los miembros de Redsicon: Estudio de caso. Revista Visión Contable, (21), 121-141. https:// doi.org/10.24142/rvc.n21a6

Chango Galarza, M. (2019). El problema metodológico de la investigación contable: Una fundamentación basada en los enfoques positivista e interpretativo. Prospectivas UTC «Revista de Ciencias Administrativas y Económicas», 2(2), 142-151. Recuperado de: http:// investigacion.utc.edu.ec/revistasutc/index.php/prospectivasutc/article/view/273

Charres, H. (2018). Triangulación: Una estrategia adecuada para las investigaciones en las ciencias administrativas y contables en la educación superior. Acción y Reflexión Educativa, (43), 152-165. Recuperado de: https://www. revistas.up.ac.pa/index.php/accion_reflexion_educativa/article/view/565

Escobar Pérez, B., \& Lobo Gallardo, A. (2014). Implicaciones Teóricas y Metodológicas de la Evolución de la Investigación en Contabilidad de Gestión. Spanish Journal of Finance and Accounting / Revista Española de Financiación y Contabilidad. Recuperado de: https://www.tandfonline.com/doi/abs/10.1080/0210241 2.2002.10779450

González-Díaz, R. R., \& Serrano Polo, E. A. (2018). Entrevistas Espontaneas Categoriales (EEC) para la construcción de categorías orientadoras en la investigación cualitativa. Journal of Latin American Science, 2(1), 1-11. Recuperado de: https://lasjournal.com/index. php/abstract/article/view/2

Gutiérrez Huby, A. M. (2005). Importancia de los indicadores en la autoevaluación de la Facultad de Ciencias Contables. Quipukamayoc, 12(24), 59-63. https:/doi. org/10.15381/quipu.v12i24.5442

Hernández Rodríguez, D. R., \& Escobar Castillo, A. E. (2017). Modelo de contabilidad social como herramienta de gestión para la responsabilidad social empresarial. IJMSOR: International Journal of Management Science \& Operation Research, 2(1), 44-56. Recuperado de: http://ijmsoridi.com/index.php/ijmsor/article/view/86

Mejía Soto, E., Morra Roa, G., \& Montes Salazar, C. A. (2013). Adscripción de la contabilidad en la estructura general del conocimiento. Cuadernos de Contabilidad, 14(34), 159-187. Recuperado de: https://dialnet.unirioja.es/servlet/articulo? codigo $=5447038$

Monsalve, K. M. (2006). Las Ciencias Contables desde el Pensamiento Complejo: El Nuevo Rol Profesional (Tesis de Maestría en Ciencias Contables). Universidad de los Andes, Mérida. Recuperado de: http://bdigital. ula.ve/storage/pdftesis/postgrado/tde_arquivos/53/ TDE-2011-07-15T05:34:10Z-1196/Publico/mejiaskarina_parte1.pdf

Moscoso Córdova, J. (2019). La investigación cualitativa y la contabilidad: El rol de los estudios en ciencias contables en la construcción de sociedades más justas y equitativas. Prospectivas UTC «Revista de Ciencias Administrativas y Económicas», 2(2), 171-184. Recuperado de: http://investigacion.utc.edu.ec/revistasutc/ index.php/prospectivasutc/article/view/289 
Naranjo Rivera, O. (2009). Aportes para un debate sobre el método de la investigación cualitativa y la investigación contable. Adversia, (4), 105-114. Recuperado de: https://revistas.udea.edu.co/index.php/adversia/ article/view/2035

Ortíz Sánchez, L. M. (2020). La teoría fundamentada como método de investigación para el desarrollo de la educación contable. Revista Visión Contable, (22), 6077. https://doi.org/10.24142/rvc.n22a3

Ortiz-Ocaña, A. L. (2016). Introducción a la investigación contable. https://doi.org/10.16925/greylit.1370

Ospina Delgado, J. E. (2018). La Investigación Cualitativa en Contabilidad: Un enfoque alternativo para comprender las prácticas contables en Colombia. En C. Barrios y W. Rojas (Eds.). Conjunciones y disertaciones: pensando la contabilidad en el siglo xxi (pp.161-177). Cali: Pontificia Universidad Javeriana.

Ospina Zapata, C. M. (2005). Sobre la investigación en contabilidad: Algunos apuntes. Contaduría Universidad de Antioquia, (46), 73-110. Recuperado de: https://revistas.udea.edu.co/index.php/cont/article/ view/25679

Pérez Stizzoli, V. (2014). La investigación cualitativa en contabilidad: Un análisis a partir de revistas indexadas. CAPIC REVIEW, 12(2), 147-158. Recuperado de: https://dialnet.unirioja.es/servlet/articulo?codigo $=7145015$
Pérez, V., \& Pinto Perry, G. R. P. (2011). Una revisión de las metodologías de investigación contable. CAPIC REVIEW, 9(1), 3.

Pinto Perry, G. R. (2010). Hacia una metodología de la investigación contable. CAPIC REVIEW, 8(1), 4. Recuperado de: https://dialnet.unirioja.es/servlet/articulo? codigo $=7144973$

Quintero Dávila, J. H. (2012). El uso del valor contable en la gerencia de las PyMES venezolanas. https://doi. org/10.26457/recein.v10i39.72

Saavedra, M., \& Saavedra, M. (2015). La investigación contable en Latinoamérica. Actualidad contable FACES, 18(31), 99-121. Recuperado de: https://www.redalyc.org/pdf/257/25743363006.pdf

Villarreal, J. L., \& Córdoba Martínez, J. X. (2017). Fundamentos para el estudio de las corrientes de investigación en contabilidad. Tendencias, 18(2), 139-151. https://doi.org/10.22267/rtend.171802.81 